\title{
Utilizing aLIGO Glitch Classifications to Validate Gravitational-Wave Candidates
}

\author{
Derek Davis $^{1,2}$, Laurel V. White ${ }^{1}$, and Peter R. Saulson ${ }^{1}$. \\ ${ }^{1}$ Syracuse University, Syracuse, NY 13244, USA \\ ${ }^{2}$ California Institute of Technology, Pasadena, CA 91125, USA
}

\begin{abstract}
Advanced LIGO data contains numerous noise transients, or "glitches", that have been shown to reduce the sensitivity of matched filter searches for gravitational waves from compact binaries by increasing the rate at which random coincidences occur. The presence of these transients has precipitated extensive work to establish that observed gravitational wave events are astrophysical in nature. We discuss the response of the PyCBC search for gravitational waves from stellar mass binaries to various common glitches that were observed during Advanced LIGO's first and second observing runs. We show how these transients can mimic waveforms from compact binary coalescences and quantify the likelihood that a given class of glitches will create a trigger in the search pipeline. We explore the specific waveform parameters that are most similar to different glitch classes and demonstrate how knowledge of these similarities can be used when evaluating the significance of gravitational-wave candidates.
\end{abstract}




\section{Introduction}

Advanced LIGO's (aLIGO) first and second observing runs (O1 and O2) have allowed gravitational waves to be detected for the first time. During these obsevring runs, aLIGO observed gravitational-wave signals from at least 10 binary black hole (BBH) systems [1] and 1 binary neutron star (BNS) system [2].

The detection of the first BNS, GW170817, was initially complicated by the presence of a loud instrumental noise transient that had to be removed before analysis could be completed [2,3]. While a somewhat unlikely coincidence, this event highlighted the nature of aLIGO data as neither Gaussian nor stationary over long time periods $[4,5,6]$. The presence of noise transients, generally referred to as "glitches", have been shown to impact the sensitivity of searches for gravitational waves from compact binary coalescence (CBC) by mimicking the appearance of a gravitational wave to matched filter based searches $[6,7,8]$. When a candidate signal is identified by a search pipeline, rigorous studies can be undertaken regarding the time in question to understand if the trigger is related to instrumental causes $[1,9,10]$. Tests to quickly evaluate the data quality around a candidate signal before initiating an additional search for an electromagnetic (EM) counterpart were routinely completed as part of the O2 EM follow up process [11].

Previous work on detector characterization of gravitational-wave interferometers $[1,6,12,13,14,15,16]$ has identified a number of classes of glitches that are present in the data. These classifications have allowed valuable follow up using auxiliary sensors $[9,10,17,18,19,20,21]$ to identify causes of each class and improve the overall gravitational-wave data quality. If a known glitch class can be associated with a well understood witness sensor, mitigation of the glitch may be possible either through instrumental intervention or by identifying these periods of data as corrupted. Unfortunately, there is a number of identified classes of glitches for which mitigation methods are not yet understood. For these glitch classes, understanding how searches can separate instrumental transients from similar astrophysical signals is the highest priority.

This paper demonstrates how the most distinguishable glitch classes affect matched filter searches for gravitational waves from CBC sources and how to evaluate the significance of gravitational-wave candidates near glitches. In order to identify periods that are corrupted by known classes of glitches, we take advantage of Gravity Spy, a machine-learning-based image classifier [12]. We examine the response to these glitches by the PyCBC search pipeline, one of the pipelines used to find $\mathrm{CBC}$ signals with aLIGO [22, 23, 24]. We show how these glitches can mimic waveforms from astrophysical sources of gravitational waves and quantify the likelihood of a given glitch from each glitch class to create a significant trigger in PyCBC. We then demonstrate how measurements of the background during times coincident with glitches can be used to evaluate if candidate signals are consistent with the expected response of the search to a population of glitches.

\section{PyCBC Detection Statistic}

In this work we utilize the PyCBC [22, 23, 8] search pipeline as an example matched filter search for CBC signals. While this work may be broadly applicable to other

analysis pipelines used to identify CBC signals with matched filtering [25, 26], differences in how pipelines rank candidates may result in slight differences in the 
effect of glitches on the analysis. An overview of how PyCBC identifies and ranks significant candidate triggers follows.

The PyCBC search pipeline is designed to identify gravitational waves generated by compact binary coalescences in interferometer data. To evaluate the significance of an individual candidate, each trigger is ranked based on the PyCBC detection statistic. While the complete PyCBC detection statistic is designed for identifying triggers that are coincident between detectors, we consider primarily the single detector detection statistic. This has three components: the signal-to-noise ratio (SNR), the chi-squared $\left(\chi^{2}\right)$ discriminator, and the sine-Gaussian discriminator.

The core of the detection statistic is the signal-to-noise ratio for a given template. The SNR for a matched filter with a specific waveform template $h$ is

$$
\rho^{2} \equiv \frac{\|\langle s \mid h\rangle\|^{2}}{\langle h \mid h\rangle},
$$

where the inner product is given by

$$
\langle a \mid b\rangle=4 \int_{0}^{\infty} \frac{\tilde{a}(f) \tilde{b}^{*}(f)}{S_{n}(f)} d f,
$$

with $s$ the strain data and $S_{n}(f)$ the measured power spectral density for the time in question.

If aLIGO noise was perfectly Gaussian, the matched filter SNR alone would be the optimal detection statistic. However, since the data contains non-Gaussian fluctuations [6, 7], numerous additional signal consistency tests are required to discriminate between instrumental artifacts and astrophysical signals. In an ideal signal consistency test, noise triggers are assigned a lower ranking statistic than comparably loud astrophysical signals.

To provide this discriminatory power, one of the most useful tests for gravitational-wave signals is the chi-squared discriminator [27]. The test is constructed by dividing the frequency space spanned by the waveform template into bins of equal power, and checking if each bin contributes the expected amount of power. This gives a measure of how well a candidate trigger matches the signal morphology of the template. Specifically, the chi-squared discriminator for a trigger is given by

$$
\chi_{r}^{2}=\frac{1}{2 p-2} \sum_{i=1}^{p}\left\|\left\langle s \mid h_{i}\right\rangle-\left\langle h_{i} \mid h_{i}\right\rangle\right\|^{2},
$$

with $h_{i}$ the waveform template in a given frequency bin.

This value should follow a reduced $\chi^{2}$ distribution with $2 p-2$ degrees of freedom. The choice of $p$ is scaled based on the duration of each template, so that a sufficient number of bins with measurable power are used. If the value of the chi-squared test is greater than unity, the detection statistic for the related trigger is reduced to produce a "re-weighted SNR", $\tilde{\rho}$. This is

$$
\tilde{\rho}=\left\{\begin{array}{ll}
\rho & \text { for } \chi_{r}^{2} \leq 1 \\
\rho\left[\frac{1}{2}\left(1+\left(\chi_{r}^{2}\right)^{3}\right)\right]^{-1 / 6} & \text { for } \chi_{r}^{2}>1
\end{array} .\right.
$$

The effectiveness of the chi-squared discriminator has been shown to be dependent on the duration of the signal and the number of bins used in the test [7]. For long 
duration signals, the test provides excellent rejection of many classes of glitches. For short duration signals, this test has reduced efficiency. To help address this effect for short duration templates, an additional signal consistency test, the sine-Gaussian discriminator, is utilized.

The sine-Gaussian discriminator is designed to downrank triggers with excess power at frequencies above the expected maximal frequency of the signal at merger [8]. If excess power is detected above this frequency, the trigger is not likely to be generated by a CBC signal. To quantify the excess power present at high frequencies, a number of sine-Gaussian wavelets with frequencies above this maximum are matched filtered against the data. These wavelets are parameterized by their frequency, $f_{0}$, central time $t_{0}$, and quality factor $Q$. In the time domain, each wavelet can be written as

$$
g(t)=\exp \left(-4 \pi f_{0}^{2} \frac{\left(t-t_{0}\right)^{2}}{Q^{2}}\right) \cos \left(2 \pi f_{0} t+\phi_{0}\right) .
$$

A new signal discriminator can be written down as the sum of the measured matched filter SNR squared of each individual sine-Gaussian tile. In the case of $N$ different tiles, this is

$$
\chi_{r, s g}^{2} \equiv \frac{1}{2 N} \sum_{i=1}^{N} \rho_{i}^{2}=\frac{1}{2 N} \sum_{i=1}^{N}\left\langle s \mid \tilde{g}_{i}\left(f, f_{0}, t_{0}, Q\right)\right\rangle^{2} .
$$

Similar to the chi-squared discriminator, this statistic should follow a reduced $\chi^{2}$ distribution with $2 N$ degrees of freedom for astrophysical signals. The result of this test is then used to compute a new detection statistic, $\tilde{\rho}_{s g}$, defined as

$$
\tilde{\rho}_{s g}=\left\{\begin{array}{ll}
\tilde{\rho} & \text { for } \chi_{r, s q}^{2} \leq 4 \\
\tilde{\rho}\left(\chi_{r, s q}^{2} / 4\right)^{-1 / 2} & \text { for } \chi_{r, s q}^{2}>4
\end{array} .\right.
$$

The value of 4 (as opposed to 1) is chosen as the threshold to account for the expected variability of $\tilde{\rho}_{s g}$ in Gaussian noise. Values above 4 are indicative of likely nonGaussian features in the data.

Even with these consistency tests, many glitches still produce significant triggers in the search. Extensive investigation is done to identify periods corrupted by problematic glitches and and remove these time segments from the analysis [7]. However, these analyses are primarily done with auxiliary witness sensors, and do not rely upon the gravitational-wave strain data to identify glitches. One of the reasons for this current lack of investigations based on analyses of the strain data is the need for

a robust classification method for glitches that is not based on the matched filtering pipeline itself.

\section{Gravity Spy Classification}

In order to classify glitches in this work, we use Gravity Spy [12, 28], a machine learning based classification tool that utilizes citizen science efforts. Gravity Spy has been used to quantify glitch rates and identify large sets of similar glitches [29, 30, 31, 32], as it can quickly and accurately identify common classes of instrumental artifacts in the detector. These studies have generally been aimed at understanding detector performance and the sources of these glitch classes. However, Gravity Spy can also be 


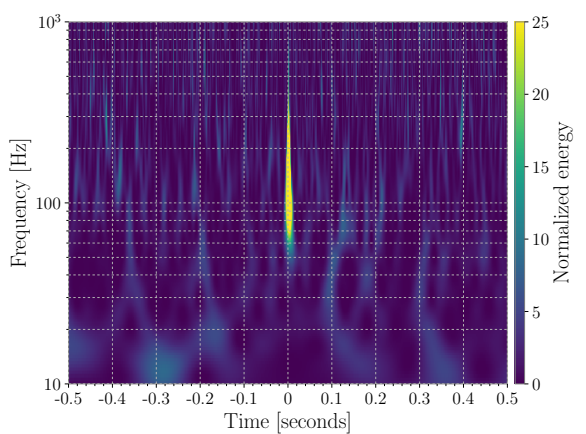

(a) Blips

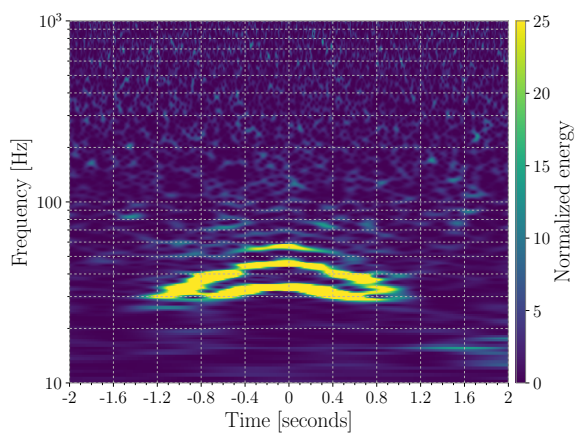

(c) Scattered Light

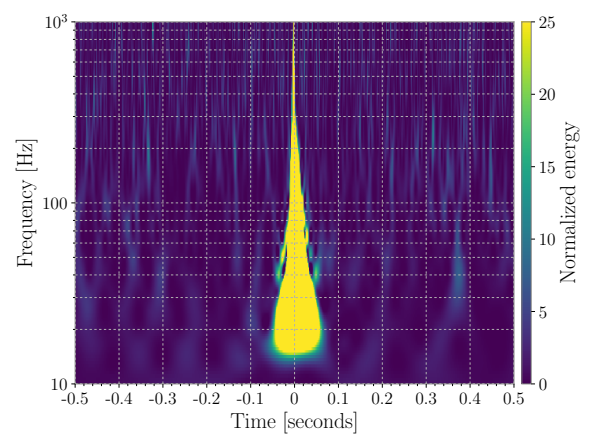

(b) Koi Fish

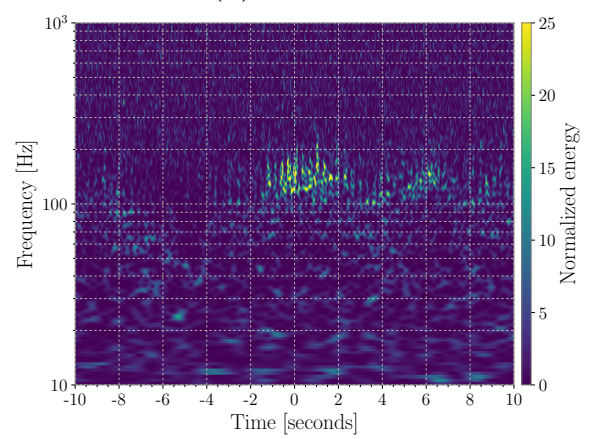

(d) Scratchy

Figure 1: Spectrograms of the four problematic Gravity Spy glitch classes discussed in this work. The glitch classes blips (top-left), koi fish (top-right), scattered light (bottom-left) and scratchy (bottom-right) are highlighted here due to their known impact on the PyCBC search. Note the diversity in duration and morphology among these four glitch classes.

used to study the impact of glitches on searches for gravitational waves as it provides a method to develop an initial dataset of glitches to investigate that is independent of the search methodology. This section explains the data selection process for the Gravity Spy pipeline including possible selection effects relevant to this study. Full details on the classification methods for Gravity Spy can be found in [12].

At its core, Gravity Spy is an image classifier based on convolutional neural network methods [33, 34]. Before the classifier can be applied, time periods containing glitches must be identified and the relevant detector data translated into an image format that the neural network can process. To identify a glitch, the Gravity Spy pipeline takes advantage of the Omicron pipeline [35]. Omicron uses a set of sineGuassian wavelets to identify excess power in detector data. Any Omicron trigger with an SNR above 7.5 is reported to the pipeline. Once a time window containing a glitch or astrophysical signal is identified, the time series data is transformed into a spectrogram using the Q-transform [36]. This representation provides the input that both the machine learning classifier and citizen scientists will use in their classification efforts. This glitch image is fed into the classifier and a confidence score ranging from 0.0 to 1.0 is given for each category. The total sum over all categories is 1.0, with the highest numeric value representing the most likely classification. 
The SNR threshold from Omicron is used to increase the chance that a clearly defined glitch will be visible in the spectrogram representation and decrease the overall size of the dataset. In the context of using these classifications for understanding the effect of glitches on search pipelines, this does provide some bias, as noise sources problematic to the searches may not meet this threshold. This consideration is especially important for long duration signals that are not expected to be identifiable in this representation, but can be found using matched filtering.

Another important consideration is the set of possible classifications that Gravity Spy can provide. While the list of possible classifications the pipeline can assign includes "None of the Above" and "No Glitch" classes, the classification is mostly limited to predetermined classes from a training set [37, 28]. Therefore, if a glitch unknown to the pipeline is classified, the result has a much higher chance of being incorrect, thus contaminating the glitch set. To guard against this problem, we set a minimum confidence of 0.95 for all glitch classes to reduce the risk of contamination.

Of the classes Gravity Spy has in its training set, we will focus on four in this work: "blip", "koi fish", "scattered light", and "scratchy". spectrograms of representative examples of each of these glitches are shown in Figure 1. These four are chosen as they have been previously identified as problematic for searches for gravitational waves from compact binaries $[1,7]$. These glitches are also some of the most common glitches in the LIGO detectors, allowing for a broad statistical study. Finally, each of these classes has yet to be completely mitigated via instrumental means in the LIGO detectors. Due to this, it is likely these glitches will be present in future observing runs and continue to limit the sensitivity of searches. Further discussion of these glitch classes can be found in $[1,7,6]$.

\section{How different kinds of glitches mimic traits of signals}

PyCBC signal consistency tests have been shown to discriminate well between glitches and astrophysical signals $[7,8,22,23,27]$. However, the wide range of template parameters included in the search [38], combined with the wide variety of instrumental artifacts, means that this discriminating power is not uniformly effective across the entirety of the search parameter space [7].

This concern is easily demonstrated for blip glitches. When plotted against a timeseries of the data around a representative blip glitch, there is significant overlap similarity between a blip and a the best matching template in the PyCBC bank, as shown in Figure 2. A blip waveform has a few short, loud cycles, similar to a sineGaussian pulse $[6,8,21]$. The detector is less sensitive at lower frequencies, meaning a template that reaches merger by $100 \mathrm{~Hz}$ will be in the observable band for only a few gravitational wave cycles and qualitatively match the model for a representative blip. Such templates correspond to some of the most massive systems in the PyCBC template bank. As blip glitches do not occur coincident in both detectors, they are known to be instrumental artifacts. However, these glitches have been noted as one of the limiting sources of noise for searches for gravitational waves from high mass binary black holes $[39,40]$.

To identify PyCBC triggers coincident with a glitch, we analyzed short segments of data around glitches identified by Gravity Spy as belonging to a specific glitch class, and recorded all PyCBC triggers that met a minimum detection statistic value. Multiple seconds of data after the glitch were included to ensure that the full duration of the glitch was included and that triggers intersecting the inspiral component of 


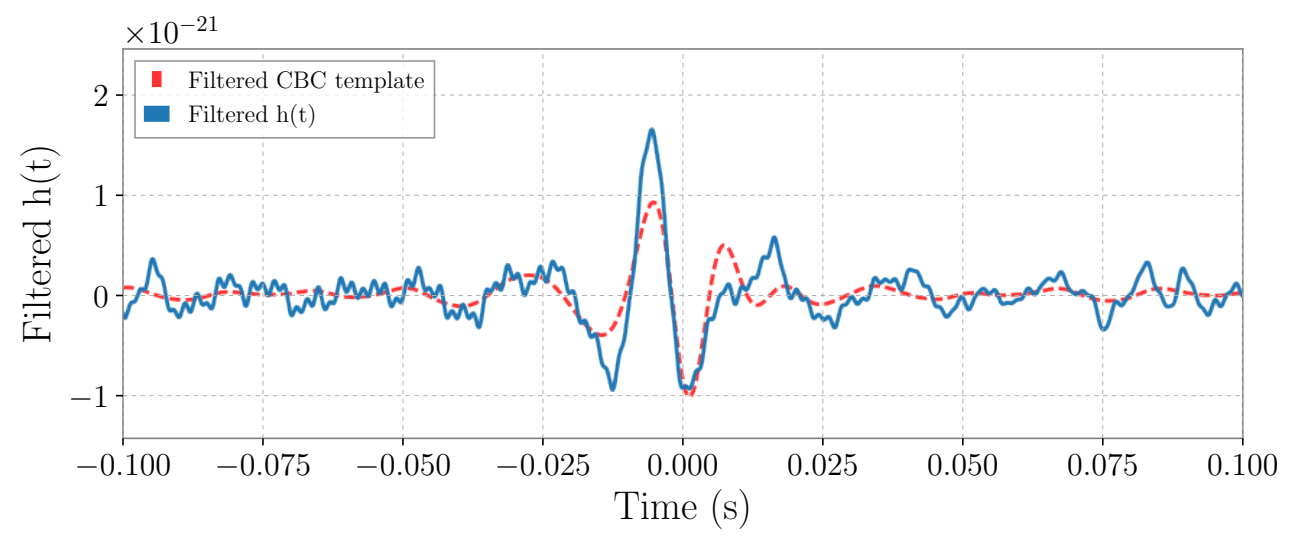

Figure 2: An overlay of a timeseries of a specifically chosen high-mass CBC template and detector data around a blip glitch. Both of the timeseries have been filtered with bandpass filters to isolate the most sensitive region of $35-500 \mathrm{~Hz}$ and notch filters to repress noise from calibration lines and harmonics of the 60 $\mathrm{Hz}$ power line. This visualization serves to show the similarity between a blip glitch and a CBC template after the response of the detector is considered.

the waveform were correctly recorded. This duration was different for each glitch class; a 2 second window was used for blips and koi fish, 4 seconds for scattered light, and 30 seconds for scratchy. In cases when the time window for neighboring glitches intersected, this was considered one glitch. These single-detector PyCBC triggers that are idenitifed coincident with Gravity Spy glitches are used throughout this work to explore how glitches can mimic CBC signals. Using this set of PyCBC triggers during times identified by Gravity Spy, we examine which CBC templates are most likely to give a significant false positive to each kind of glitch.

For each class of glitch in our data set, we test to see the likelihood that a single glitch from this class produces a trigger above a detection statistic threshold (in this case $\tilde{\rho}_{s g}>7.0$ in each bin) in each part of the template bank parameter space. This value was chosen because a trigger of this detection statistic value combined with the minimal value possible in the other detector $\left(\tilde{\rho}_{s g} \approx 5.5\right)$ would result in a candidate with a combined network detection statistic of $\tilde{\rho}_{\text {sg,net }}>9.0$, strong enough to be identified separate from the background at a false alarm rate of approximately 1 per year. We choose to bin the parameter space spanned by the O2 PyCBC template bank uniformly in both $\chi_{\text {eff }}$ (the effective spin of the system) and $\log \left(M_{\text {total }}\right)$ to help account for the reduced density of templates at high $M_{\text {total }}$. The probability of a glitch producing a trigger in each bin is highly dependent on the total number of templates within the bin, and hence the size of the bin itself. However, the regions of the parameter space that are identified as the most likely to produce a trigger are robust to choice of bin size.

In each template bank bin, we calculate the fraction of glitches in a given class that produced at least one trigger above the chosen threshold that have parameters consistent with the bin in question. Since each bin probability is calculated independently, the sum over all bins is not bounded above by 1 . In fact, it is possible for a given glitch instance to create triggers recorded in multiple bins. This allows the full extent of the glitch overlap with the template bank to be recorded. The results 


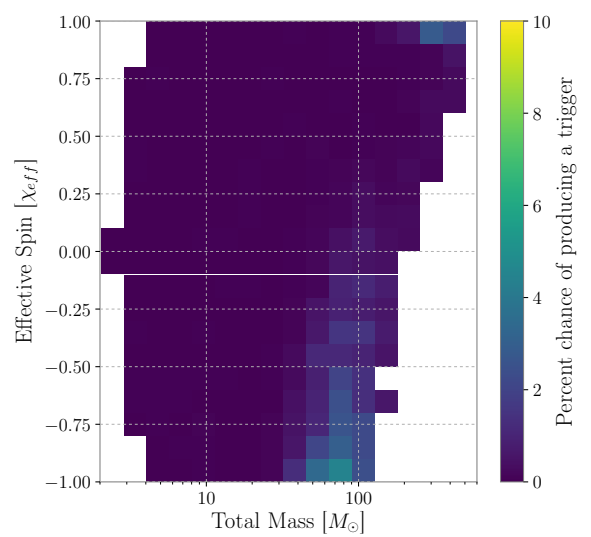

(a) Blips

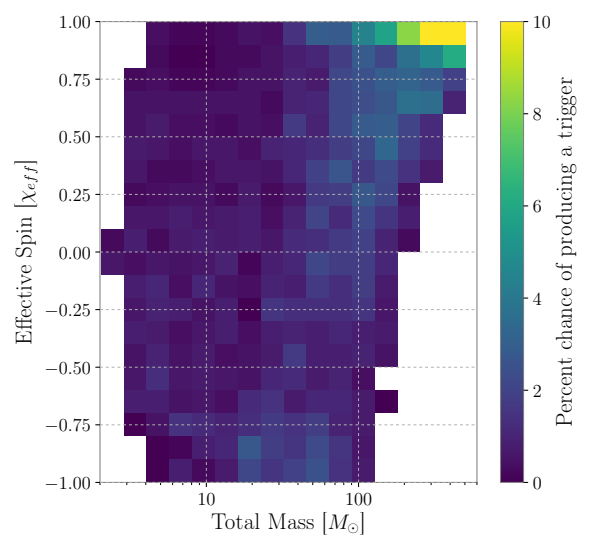

(c) Scattered Light

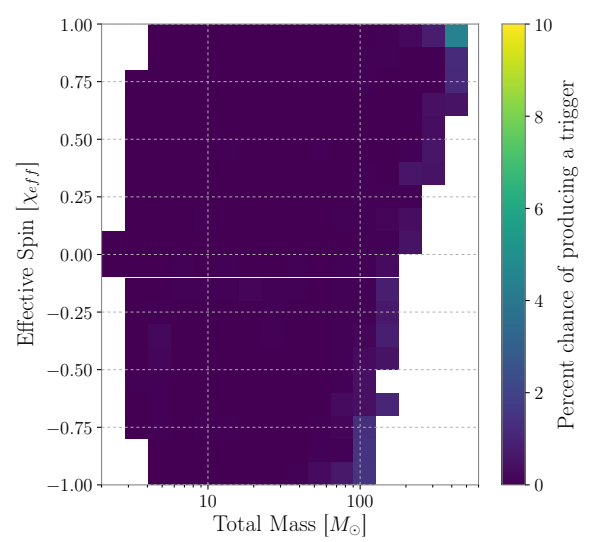

(b) Koi fish

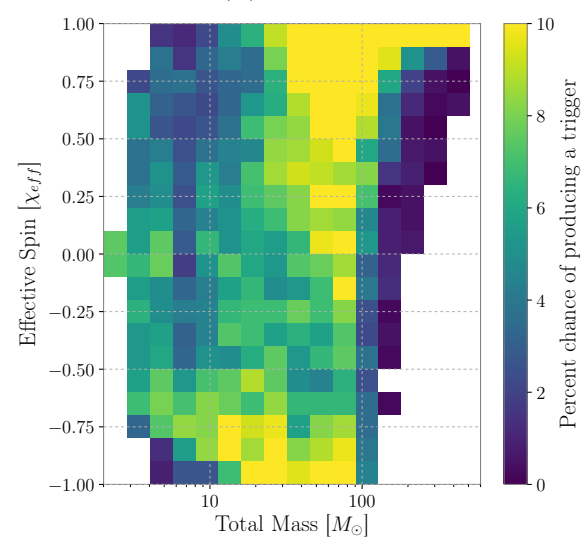

(d) Scratchy

Figure 3: Probability of producing a trigger above sine-Gaussian SNR of 7.0 in specific regions of the template bank parameter space for each glitch class. Triggers are binned by total mass and effective spin of the corresponding template. Blips (top-left), koi fish (top-right), scattered light (bottom-left) and scratchy (bottom-right) each show maximum probabilities in different parts of the parameter space.

of this study can be seen in Figures 3 and 4 for the four glitch classes considered in this work and for all of the template bank bins. Figure 3 has templates binned by total mass $\left(M_{t o t}\right)$ and effective spin $\left(\chi_{e f f}\right)$ while Figure 4 has templates binned by component mass.

Blip glitches show a strong clustering at the highest total mass in the template bank and maximally anti-aligned effective spins. In terms of component masses, the highest probability regions correspond to a primary mass of approximately $100 \mathrm{M} \odot$ and a smaller secondary mass. Notably, the highest probability for any region of the template bank is below 5\%. It is important to note that blips are one of the most common classes of instrumental transient found in the detectors, so the low probability shown here may still impact the sensitivity of the search. The rate of blip glitches is 1-2 per hour [1], meaning that this rate corresponds to almost one significant blip-related 


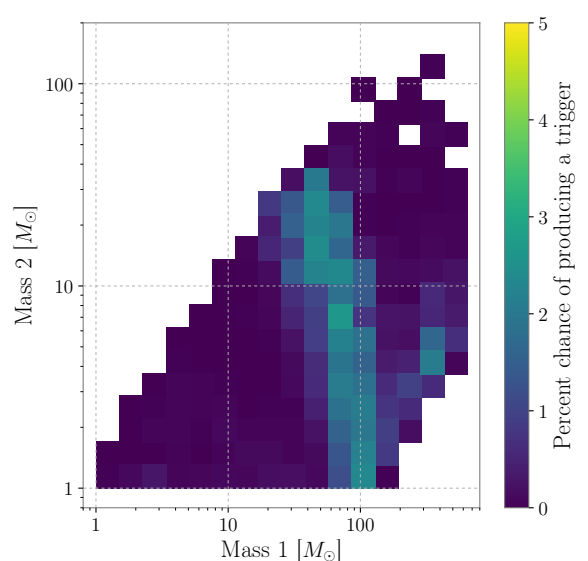

(a) Blips

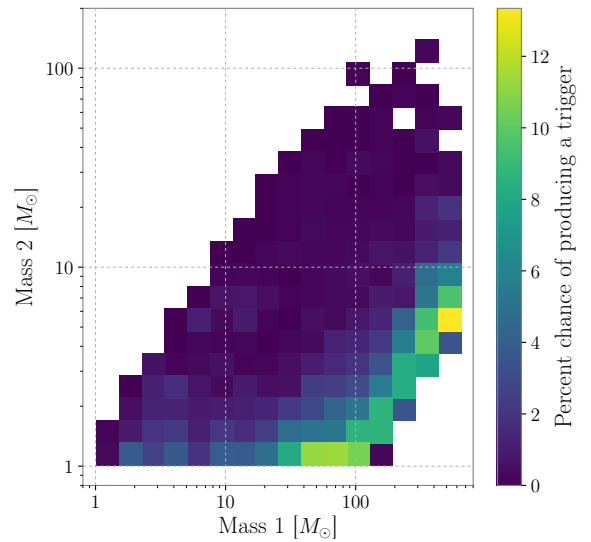

(c) Scattered Light

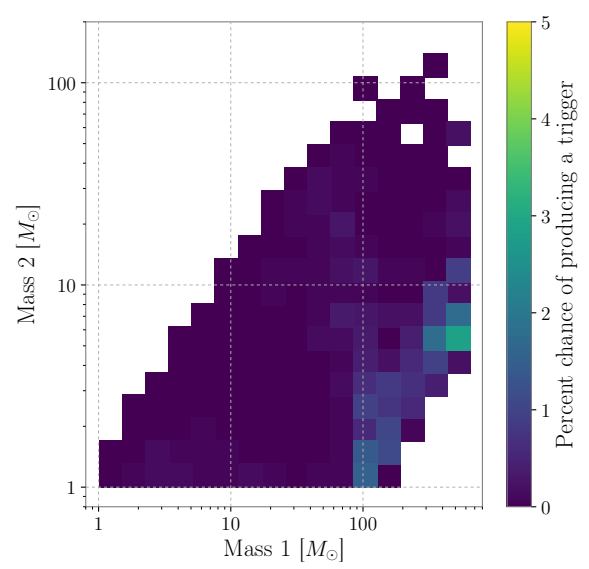

(b) Koi Fish

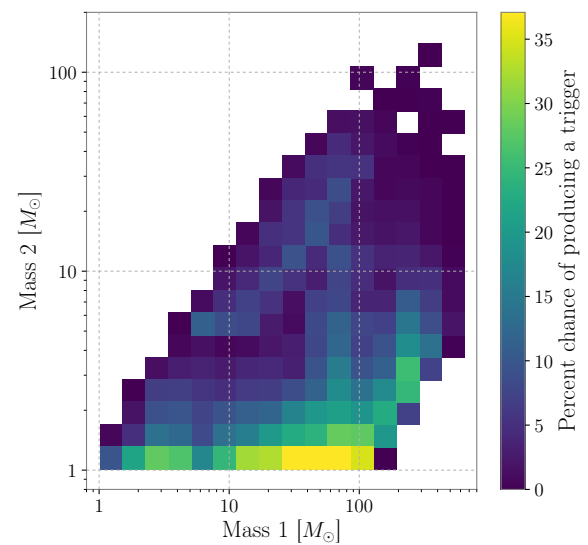

(d) Scratchy

Figure 4: Probability of producing a trigger above sine-Gaussian SNR of 7.0 in specific regions of the template bank parameter space for each glitch class. Triggers are binned by component masses of the corresponding template. Blips (top-left), koi fish (top-right), scattered light (bottom-left) and scratchy (bottom-right) each show maximum probabilities in different parts of the parameter space. Note the differing range of values for each glitch class.

trigger each day.

Triggers from koi fish glitches are most likely to be found with high component masses, and maximal spins, both aligned and anti-aligned. Similar to blip glitches, these triggers correspond to short duration templates. However, koi fish triggers do not show the high probability cluster for maximally anti-aligned templates found for blip triggers.

The highest probability region for scattered light glitches is short duration highly aligned spin templates. These templates experience a 'hang-up' effect [41], resulting in a template that can match the arch-like morphology of a typical scattered light glitch. Note that there is a large swath of parameter space that has a noticeable response to a typical scattered light glitch. This is likely a result of the large number of sources of 
scattered light in the interferometer, which may produce differing glitch morphologies.

Triggers coincident with scratchy glitches overlap well with a large range of templates, with the highest probability clusters for maximally aligned and anti-aligned spins and total mass above $20 M_{\odot}$. Projecting this result onto the component mass parameter space shows that this region also corresponds to templates with high mass ratios, such as those from neutron star - black hole (NSBH) systems. In addition to high mass triggers, the component mass parameter space also shows a high probability of producing a trigger with component masses of $3 M_{\odot}$ and $1 M_{\odot}$. While scratchy glitches are rarer than the other classes identified in this work, the likelihood of a single glitch producing a significant trigger is high for a wide range of parameters, a contrasting situation to blips and koi fish. This is partially due to the typical timescale of a scratchy glitch, lasting up to multiple minutes, as opposed to Blip and koi fish glitches that last tenths of seconds. While this does increase the chance of a trigger being due to chance, the excess is significantly higher than we would expect from colored Gaussian noise alone. Scratchy glitches are also the only class of the four surveyed in this work that are shown to have a high probability of producing a significant trigger with a total mass below $20 M_{\odot}$.

These results on their own can not preclude the possibility of a genuine signal to occur in coincidence with a glitch (as was the case for GW170817). However, for each glitch class, there does appear to be a range of parameters for which a candidate trigger in time coincidence would likely be caused by the presence of the glitch. This clustering allows these results to be used to quickly follow up candidates after they are identified by the search to understand if the recovered parameters are likely to be produced by the glitch in question.

For example, if a significant gravitational-wave candidate was identified during a time period classified by Gravity Spy as a blip, the candidate trigger is unlikely to be related to the observed instrumental artifact unless trigger is a high mass, anti-aligned template. Conversely, a trigger candidate coincident with a glitch that has parameters consistent with a high probability region of the glitch would warrant additional investigations to understand a possible connection between the artifact and candidate trigger

Notably, the mass and spin parameters of previously observed BBH and BNS signals [1] are not consistent with any of the highest probability regions for any of the four glitch classes examined in this work. This suggests that it unlikely for any of these common glitch classes to mimic a signal from the currently observed gravitational-wave population.

\section{Utilizing glitch classification in significance estimates}

As discussed in the previous section, the known correlations between specific template parameters and glitch classes do not preclude the possibility of a real astrophysical signal occurring in coincidence with a glitch. In this scenario, it may be possible to include the additional information we have about the expected overlap with the candidate trigger parameters and the glitch population to re-evaluate the significance

of the candidate. In this section we outline a procedure to calculate the significance of a trigger found in time coincidence with a time classified by Gravity Spy as belonging to a specific glitch class. Rather than producing a "yes or no" result on whether a gravitational-wave candidate is "caused" by the presence of a glitch, we can reestimate the significance of a candidate based on the expected rate of triggers due 

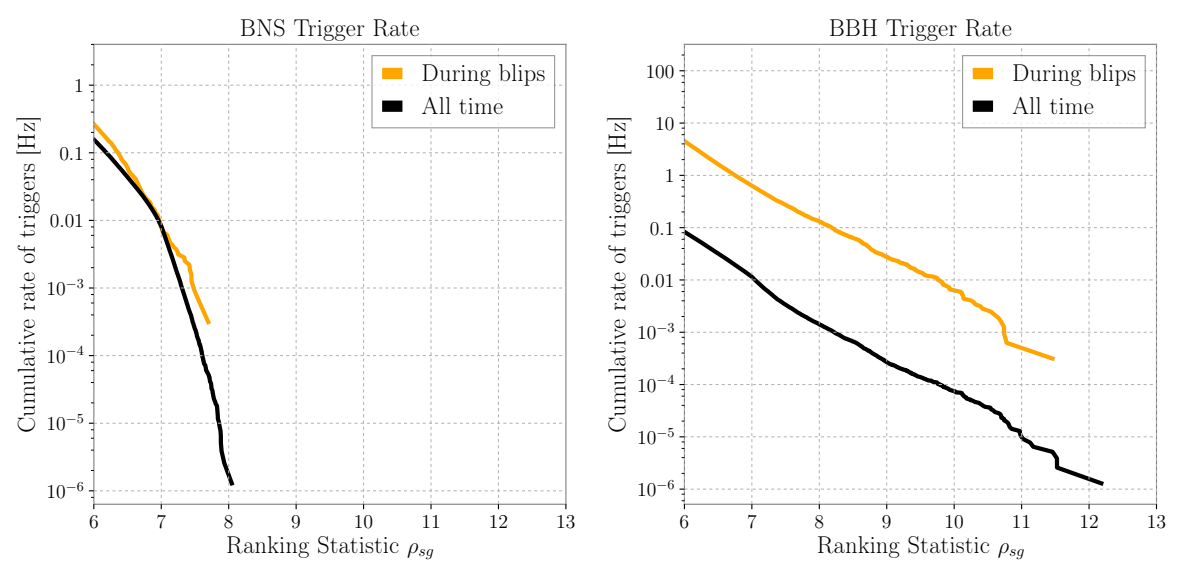

Figure 5: A comparison of the false alarm rate (FAR) based on all times in the analysis and only times coincident within 2 seconds of blip. The trigger rate is much higher during blip periods for BBH triggers, supporting the previous conclusion that there is an increased chance of producing a trigger coincident with this source of noise. Left: Difference in trigger rate for $\mathrm{BBH}$ triggers. Right: Difference in trigger rate for BNS triggers.

to the glitch. This approach decreases the risk that a astrophysical signal will be discarded due to data quality issues. We focus on blip glitches as a test case since they are one of the most common glitches in both detectors and have very defined regions of the parameter space where overlap between signals and glitches occur.

To first demonstrate how glitching changes the trigger rate in different parts of the parameter space, we compare trigger rates from the entire analysis period versus only times that are known to contain blip glitches. We then sort triggers from each period into a long duration (referred to as BNS) category $\left(M_{\text {chirp }}<2.0 M_{\odot}\right)$ and a short duration $(\mathrm{BBH})$ category $\left(M_{\text {chirp }}>5.0 M_{\odot}\right)$. A comparison of the rate of triggers versus network ranking statistic $\tilde{\rho}_{s g, n e t}$ for the entire analysis period and around blip glitches is shown in Figure 5. If we examine the trigger rate during the entire analysis period versus during time periods within 2 second of blip glitches, we see that there is indeed an increased rate of triggers at fixed ranking statistic for times around blip glitches. Furthermore, this increase is only apparent for the BBH category, with only minimal increases for the BNS category. This agrees with our expectation from the previous section that blip glitches are correlated with high mass triggers.

As was the case with GW170817, it may still be possible to detect a gravitationalwave signal during a time period corrupted by glitching. A critical component of this detection was establishing that the observed glitch could not have accounted for the BNS signal in the data $[2,3]$. In order to facilitate significance estimates of additional candidates, Gravity Spy classifications, combined with our knowledge of the overlaps between template parameters, can be used to evaluate if the candidate trigger is significant despite the association with a known class of glitch.

Blip glitches present a clear use case for this follow up. This class is known to impact only an isolated part of the parameter space. Specifically, we would expect that low mass BNS triggers would be uncorrelated, while high mass BBH triggers may be correlated to the presence of a glitch. In order to account for the expected variation 


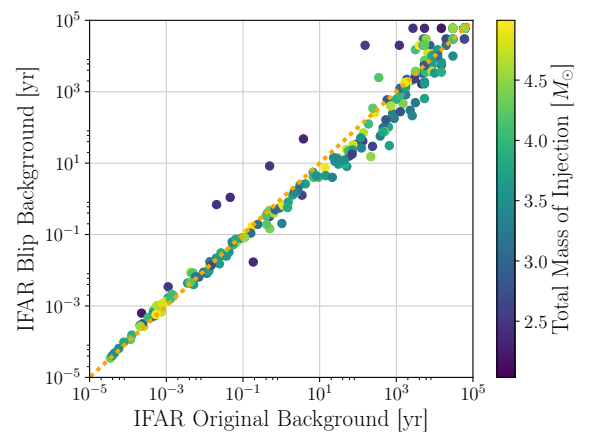

(a) BNS Injections

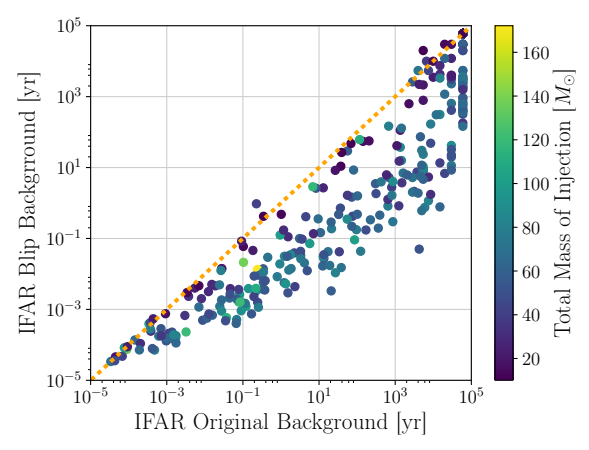

(b) BBH Injections

Figure 6: Recovered IFAR for a set of injections compared against the background from all time during an analysis (x-axis) versus the background during only blip times (y-axis). In each plot a 1-1 line is included for reference. For BNS injections (left), there is no effect on the recovered IFAR. For BBH injections (right), the high rate of triggers with similar template duration during blip times reduces the significance of the injections. This provides a natural way to evaluate a candidate trigger that is coincident with a known source of noise in the detector.

in the background distribution across the template bank, the $\mathrm{PyCBC}$ pipeline includes parameter dependent background reweighing that measures the rate of triggers with respect to template duration and downranks templates that are shown to occur more frequently [23]. As BNS and BBH signals have vastly different template durations, we would expect both classes of signals to be affected differently by the inclusion of this term to the ranking statistic when compared against our expected triggers from blips.

To demonstrate how the significance of each signal model is affected by a correlation with blip glitches, we perform a series of astrophysical software injections of both BNS and BBH signals into aLIGO data that are similar to the population of BNS and $\mathrm{BBH}$ signals previously detected by aLIGO and aVirgo. We first calculate the inverse false alarm rate (IFAR) of each injection using the background distribution measured from a 5 day analysis period as a control. We empirically measure the distribution of single detector background triggers during times period flagged by Gravity Spy as blips with confidence $>0.95$, and use this as the input for the background re-weighing procedure. We then reevaluate the IFAR of each injection with this new background distribution. A comparison of the recovered IFAR for each injection before and after including this correction based on the expected background distribution of blips can be seen in Figure 6, along with a 1-1 line indicating where the recovered IFAR is consistent between the two cases.

Comparing the two injection sets, there is a clear difference in the distribution with respect to the 1-1 line. BNS injections were recovered at approximately the same IFAR in both cases, showing that times corrupted by blips are equally likely to produce a BNS trigger as an average time. The BBH injections, on the other hand, have a clear separation from the 1-1 line. Specifically, the IFAR of injections recovered is lower when a background based on blips is used versus the current search configuration. Since blip times are more likely to produce triggers with BBH parameters, these injections are naturally downranked. However, sufficiently loud injections are still 
recovered at IFAR of greater than 1 year, which would be sufficiently significant to separate itself from the background. This allows signals which are inconsistent with the expected glitch behavior to be identified as a candidate of interest.

While only blips were studied in this section, this procedure can be repeated for all glitch classes that are sufficiently common for an expected background distribution to be measured. This method can provide further quantitative evidence that a candidate trigger is unlikely to be related to the instrumental artifact that it happens to occur in time coincidence with. In general, the presence of a glitch does not preclude the possibility that a candidate trigger is astrophysical, but only reduces the likelihood of astrophysical origin as compared to a candidate trigger that does not overlap a known instrumental artifact. As a high number of significant triggers have occurred during periods of transient noise [1], the methods outlined in this work will be able to be applied to numerous gravitational-wave events.

In addition to being used in validation procedures of significant candidates, this method can be implemented in the ranking statistic internal to the search pipeline. One possible technical solution is to separately evaluate triggers during times categorized by Gravity Spy as glitches and times where no glitch is identified. This would allow the search to benefit from an increased sensitivity during time periods where no glitching occurs, and to more accurately rank candidates related to glitching. Down ranking triggers during glitches instead of removing them from the analysis has the benefit, as compared to current data quality veto methods, of not precluding the possibility of detecting a signal during a glitch. For glitches without known witnesses, such as blips, this method also allows for Gravity Spy classifications to be incorporated into significance estimates of gravitational-wave candidates. If an astrophysical signal was classified as a glitch due to a highly similar morphology with a glitch class, this method would not prevent detection. Further studies evaluating the safety of this method, and the utilization of Gravity Spy classifications to rank candidate triggers, are warranted.

One limitation of this method is that it relies upon the specific parameters used to model the background distribution of the matched filter search. As has been shown in this work, classifying PyCBC triggers with one single variable will not be able to fully differentiate triggers due to glitches and triggers due to genuine gravitational waves. This method will likely be more effective if additional parameters are used (such as the effective spin and total mass). Detailed investigations into what parameters would be best for each glitch class may be resolved in future investigations.

\section{Discussion}

This work emphasizes how similarities between common glitches and astrophysical signals in aLIGO data can present challenges in validating gravitational-wave detections. This is especially problematic for novel sources including mergers of intermediate mass black holes [39, 42] and neutron star - black hole [43] systems. As each of these regions of the current template bank are impacted differently by each of the glitch classes, there is unlikely to be a single method to efficiently differentiate these novel sources from common instrumental artifacts. Focused work to design consistency tests that account for known problematic glitch morphologies is needed. Alternatively, developing robust mitigation techniques for each of these common glitch classes will have tangible effects on the overall sensitivity of the searches.

At the present, the quantifiable metrics developed in this work can also be used 
to guide event validation of candidate triggers. When evaluating whether to initiate a search for an electromagnetic counterpart, being able to predict whether a significant trigger is likely due to the presence of a common glitch will allow more informed and prompt alert updates. As many EM counterparts to a gravitational wave signal occur within minutes of merger [44], quick follow up is critical. Gravity Spy classifications are currently utilized in automated follow up in LIGO-Virgo's third observing run and the results of this study can be used to translate these classifications into easily used metrics to determine the likelihood of the candidate being related to a common glitch. Since the regions where a glitch is most likely to produce a trigger correspond with regions where a lower event rate is expected, understanding if a candidate of interest is a rare astrophysical signal or a common glitch is especially important to guide astronomical observations.

Once aLIGO reaches design sensitivity and the rate of detections increases, signals found near noise transients in the data will become a much more common situation. Already in the recent results from $\mathrm{O} 2$, a significant fraction of marginal triggers have been identified in time coincidence with noise transients [1]. This trend has continued during the third observing run, where a number of open alert candidates have been retracted due to data quality concerns [45, 46, 47]. Future progress in addressing these issues will be facilitated both by continual instrumental work to the reduce the rate of glitches as well as by further studies of how to distinguish glitches from genuine signals.

\section{Acknowledgments}

We would like to thank the Gravity Spy team and the Detector Characterization group for identifying and characterizing the noise sources addressed in this work, as well as the PyCBC search group for development of the pipeline. We also thank Scott Coughlin, Joshua Smith, and Thomas Massinger for helpful discussions. Computing support for this project was provided by the LDAS computing cluster at the California Institute of Technology.

DD, LW, and PRS acknowledge support from NSF award PHY-1607169. LIGO was constructed by the California Institute of Technology and Massachusetts Institute

of Technology with funding from the National Science Foundation, and operates under cooperative agreement PHY-0757058. This work carries LIGO Document number P1900372.

\section{References}

[1] B. P. Abbott et al. GWTC-1: A Gravitational-Wave Transient Catalog of Compact Binary Mergers Observed by LIGO and Virgo during the First and Second Observing Runs. Phys. Rev. X, 9:031040, Sep 2019.

[2] B. P. Abbott et al. GW170817: Observation of Gravitational Waves from a Binary Neutron Star Inspiral. Phys. Rev. Lett., 119:161101, 2017.

[3] C. Pankow et al. Mitigation of the instrumental noise transient in gravitational-wave data surrounding GW170817. Physical Review D, 98(8):084016, 2018.

[4] M. Walker et al. Identifying correlations between LIGO's astronomical range and auxiliary sensors using lasso regression. Classical and Quantum Gravity, 35(22):225002, 2018.

[5] L. K. Nuttall et al. Improving the Data Quality of Advanced LIGO Based on Early Engineering Run Results. Class. Quantum Grav., 32(24):245005, 2015.

[6] B. P. Abbott et al. Characterization of transient noise in Advanced LIGO relevant to gravitational wave signal GW150914. Class. Quant. Grav., 33(13):134001, 2016. 
[7] B. P. Abbott et al. Effects of data quality vetoes on a search for compact binary coalescences in Advanced LIGO's first observing run. Classical and Quantum Gravity, 35(6):065010, 2018.

[8] A. H. Nitz. Distinguishing short duration noise transients in LIGO data to improve the PyCBC search for gravitational waves from high mass binary black hole mergers. Classical and Quantum Gravity, 35(3):035016, 2018.

[9] L. K. Nuttall. Characterizing transient noise in the LIGO detectors. Philosophical Transactions of the Royal Society of London A: Mathematical, Physical and Engineering Sciences, 376(2120), 2018.

[10] B. K. Berger. Identification and mitigation of Advanced LIGO noise sources. Journal of Physics: Conference Series, 957(1):012004, 2018.

[11] B. P. Abbott et al. Low-latency gravitational-wave alerts for multimessenger astronomy during the second advanced LIGO and virgo observing run. The Astrophysical Journal, 875(2):161, apr 2019

[12] M. Zevin et al. Gravity Spy: integrating advanced LIGO detector characterization, machine learning, and citizen science. Classical and Quantum Gravity, 34(6):064003, 2017.

[13] J. Powell et al. Classification methods for noise transients in advanced gravitational-wave detectors. Classical and Quantum Gravity, 32(21):215012, Oct 2015.

[14] J. Powell et al. Classification methods for noise transients in advanced gravitational-wave detectors II: performance tests on advanced LIGO data. Classical and Quantum Gravity, 34(3):034002, Jan 2017.

[15] N. Mukund, S. Abraham, S. Kandhasamy, S. Mitra, and N. S. Philip. Transient classification in LIGO data using difference boosting neural network. Phys. Rev. D, 95:104059, May 2017.

[16] S. Mukherjee, R. Obaid, and B. Matkarimov. Classification of glitch waveforms in gravitational wave detector characterization. Journal of Physics: Conference Series, 243:012006, aug 2010.

[17] J. R. Smith et al. A hierarchical method for vetoing noise transients in gravitational-wave detectors. Classical and Quantum Gravity, 28(23):235005, 2011.

[18] Reed Essick, Salvatore Vitale, Erik Katsavounidis, Gabriele Vedovato, and Sergey Klimenko. Localization of short duration gravitational-wave transients with the early advanced LIGO and Virgo detectors. Astrophys. J., 800(2):81, 2015.

[19] P. Ajith et al. Instrumental vetoes for transient gravitational-wave triggers using noise-coupling models: The bilinear-coupling veto. Phys. Rev. D, 89:122001, Jun 2014.

[20] T. Isogai and the LIGO Scientific Collaboration. Used percentage veto for LIGO and Virgo binary inspiral searches. Journal of Physics: Conference Series, 243:012005, aug 2010.

[21] M. Cabero et al. Blip glitches in Advanced LIGO data. Classical and Quantum Gravity, 2019.

[22] S. A. Usman et al. The PyCBC search for gravitational waves from compact binary coalescence. Classical and Quantum Gravity, 33(21):215004, 2016.

[23] A. H. Nitz, T. Dent, T. Dal Canton, S. Fairhurst, and D. A. Brown. Detecting binary compactobject mergers with gravitational waves: Understanding and improving the sensitivity of the PyCBC search. The Astrophysical Journal, 849(2):118, nov 2017.

[24] A. Nitz, I. Harry, D. Brown, C. M. Biwer, J. Willis, et al. ligo-cbc/pycbc: Post-O2 release 5, Feb 2018.

[25] C. Messick et al. Analysis framework for the prompt discovery of compact binary mergers in gravitational-wave data. Phys. Rev. D, 95:042001, Feb 2017.

[26] T. Venumadhav, B. Zackay, J. Roulet, L. Dai, and M. Zaldarriaga. New search pipeline for compact binary mergers: Results for binary black holes in the first observing run of Advanced LIGO. Phys. Rev. D, 100:023011, Jul 2019.

[27] B. Allen, W. G. Anderson, P. R. Brady, D. A. Brown, and J. D. E. Creighton. FINDCHIRP: An algorithm for detection of gravitational waves from inspiraling compact binaries. Phys. Rev. D, 85:122006, 2012.

[28] S. Coughlin et al. Updated Gravity Spy Data Set, 2018. doi: 10.5281/zenodo.1486046.

[29] Sara Bahaadini, Neda Rohani, Scott Coughlin, Michael Zevin, Vicky Kalogera, and Aggelos K Katsaggelos. Deep Multi-view Models for Glitch Classification. 2017.

[30] S. Coughlin, S. Bahaadini, N. Rohani, M. Zevin, O. Patane, M. Harandi, C. Jackson, V. Noroozi, S. Allen, J. Areeda, M. Coughlin, P. Ruiz, C. P. L. Berry, K. Crowston, A. K. Katsaggelos, A. Lundgren, C. Østerlund, J. R. Smith, L. Trouille, and V. Kalogera. Classifying the unknown: Discovering novel gravitational-wave detector glitches using similarity learning. Phys. Rev. D, 99:082002, Apr 2019.

[31] O. Patane et al. aLIGO LHO Logbook. https://alog.ligo-wa.caltech.edu/aLOG/index.php? $\mathrm{callRep}=43177$.

[32] A. Lundgren et al. aLIGO LHO Logbook. https://alog.ligo-wa.caltech.edu/aLOG/index. php?callRep=27138. 
[33] A. Krizhevsky, I. Sutskever, and G. E. Hinton. Imagenet classification with deep convolutional neural networks. In F. Pereira, C. J. C. Burges, L. Bottou, and K. Q. Weinberger, editors, Advances in Neural Information Processing Systems 25, pages 1097-1105. Curran Associates, Inc., 2012.

[34] Sara Bahaadini, Vahid Noroozi, Neda Rohani, Scott Coughlin, Michael Zevin, and Aggelos K. Katsaggelos. DIRECT: Deep Discriminative Embedding for Clustering of LIGO Data. 2018.

[35] F. Robinet. Omicron: An Algorithm to Detect and Characterize Transient Noise in Gravitational-Wave Detectors. https://tds.ego-gw.it/ql/? c=10651, 2015.

[36] S. Chatterji, L. Blackburn, G. Martin, and E. Katsavounidis. Multiresolution techniques for the detection of gravitational-wave bursts. Class. Quantum Grav., 21:S1809-S1818, 2004.

[37] S. Bahaadini, V. Noroozi, N. Rohani, S. Coughlin, M. Zevin, J.R. Smith, V. Kalogera, and A. Katsaggelos. Machine learning for gravity spy: Glitch classification and dataset. Information Sciences, 444:172 - 186, 2018.

[38] T Dal Canton and I W Harry. Designing a template bank to observe compact binary coalescences in Advanced LIGO's second observing run. 2017. arXiv:1705.01845.

[39] B. P. Abbott et al. Search for intermediate mass black hole binaries in the first observing run of Advanced LIGO. Phys. Rev. D, 96:022001, Jul 2017.

[40] BP Abbott et al. Observing gravitational-wave transient GW150914 with minimal assumptions. Phys. Rev. D, 93:122004, Jun 2016.

[41] J. Healy and C. O. Lousto. Hangup effect in unequal mass binary black hole mergers and further studies of their gravitational radiation and remnant properties. Phys. Rev. D, 97:084002, Apr 2018.

[42] J. Abadie, B. P. Abbott, R. Abbott, T. D. Abbott, M. Abernathy, T. Accadia, F. Acernese, C. Adams, R. Adhikari, C. Affeldt, and et al. Search for gravitational waves from intermediate mass binary black holes. Phys. Rev. D, 85(10):102004, May 2012.

[43] B. P. Abbott et al. Upper limits on the rates of binary neutron star and neutron star-black hole mergers from advanced LIGO's first observing run. The Astrophysical Journal Letters, 832(2):L21, 2016.

[44] K. Cannon et al. Toward early-warning detection of gravitational waves from compact binary coalescence. The Astrophysical Journal, 748(2):136, 2012.

[45] LIGO Scientific Collaboration and Virgo Collaboration. GCN, 24591, 2019. https://gcn.gsfc . nasa.gov/gcn3/24591.gcn3.

[46] LIGO Scientific Collaboration and Virgo Collaboration. GCN, 24655, 2019. https://gcn.gsfc. nasa.gov/gcn3/24655.gcn3.

[47] LIGO Scientific Collaboration and Virgo Collaboration. GCN, 25301, 2019. https://gcn.gsfc. nasa.gov/gcn3/25301.gcn3. 